\title{
Análise de dados do Enade e Enem: uma revisão sistemática da literatura
}

\author{
Data analysis of Enade and Enem: a systematic review of literature
}

\author{
Priscila da Silva Neves Lima ${ }^{1}$ \\ ${ }^{1}$ Universidade Federal de Goiás | Instituto de Informática \\ Goiânia | GO | Brasil. Contato: priscila.snl@gmail.com \\ http://orcid.org/0000-0003-4203-8800
}

Ana Paula Laboissière Ambrósio ${ }^{2}$

${ }^{2}$ Universidade Federal de Goiás | Instituto de Informática

Goiânia | GO | Brasil. Contato: apaula@inf.ufg.br

http://orcid.org/0000-0001-9553-2250

\author{
Deller James Ferreira ${ }^{3}$ \\ ${ }^{3}$ Universidade Federal de Goiás | Instituto de Informática \\ Goiânia |GO | Brasil. deller@inf.ufg.br \\ http://orcid.org/0000-0002-4314-494X
}

\author{
Jacques Duílio Brancher ${ }^{4}$ \\ ${ }^{4}$ Universidade Estadual de Londrina | Departamento de Computação \\ Londrina | PR | Brasil. Contato: jacques@uel.br \\ http://orcid.org/0000-0003-0990-747X
}
Resumo: No Brasil, a entidade responsável por promover estudos, pesquisas e avaliações sobre o sistema educacional é o Instituto Nacional de Estudos e Pesquisas Educacionais Anísio Teixeira (Inep); com o intuito de analisar a Educação Básica, dentre outras avaliações, o Inep aplica o Exame Nacional do Ensino Médio (Enem); para avaliar a qualidade da educação superior, o Exame Nacional de Desempenho de Estudantes (Enade). A partir das análises dos dados produzidos pela aplicação desses exames, é possível observar o desempenho tanto do estudante quanto das instituições e, assim, calcular indicadores de qualidade que, dentro de um contexto, oportunizarão decisões de melhorias do processo de ensino e aprendizagem. Este trabalho apresenta uma Revisão Sistemática da Literatura (RSL), com o intuito de identificar os objetivos e tipos de análises que têm sido feitos usando os dados dos exames. A revisão mostrou que essas análises são limitadas, geralmente usando estatística descritiva, e focam, principalmente, em dados socioeconômicos e notas dos exames.

Palavras-chave: Análise de dados educacionais. Enem. Enade.

Abstract: In Brazil, the entity responsible for promoting studies, research and evaluation of the educational system is the National Institute of Studies and Educational Research Anísio Teixeira - INEP. To analyze K-12 education, among other criteria, INEP applies the National High School Exam (ENEM). Quality evaluation of higher education includes the National Student Performance Exam - ENADE. Based on the analysis of the data produced by these exams, it is possible to observe the performance of both students and institutions to calculate quality indicators, which in a context, will support decisions that aim to improve the teaching and learning process. This paper presents a Systematic Review of Literature (RSL) to identify the types of analyzes that have been made with the exams' data. The review showed that the analysis is limited, most use descriptive statistics, and focus mainly on social-economic data and exam grades.

Key words: Analysis of educational data. Enem. Enade.

DOI: http://dx.doi.org/10.1590/S1414-40772019000100006

Recebido em: 5 de junho de 2017

Aprovado em: 12 de setembro de 2018

Este é um artigo publicado em acesso aberto (Open Access) sob a licença Creative Commons Attribution Non-Commercial, que permite uso, distribuição e reprodução em qualquer meio, sem restrições desde que sem fins comerciais e que o trabalho original seja corretamente citado. https://creativecommons.org/licenses/by-nc/4.0/ 


\section{Introdução}

Um dos principais objetivos da avaliação educacional é garantir a qualidade do ensino. Nesse contexto, entende-se avaliação como "um juízo de qualidade sobre dados relevantes, tendo em vista uma tomada de decisão" (LUCKESI, 2010). Ao realizar essa avaliação em âmbito Federal, é possível construir indicadores de qualidade que servem para valorar políticas, programas, projetos e ações educativas (FERREIRA; TENÓRIO, 2010). A partir das análises dos dados educacionais é possível observar o desempenho tanto do estudante quanto das instituições, e, assim, calcular indicadores de qualidade, que, dentro de certo contexto, oportunizarão decisões de melhorias ao processo de ensino e aprendizagem.

Para avaliar o ensino superior foi estabelecido, em 2004, o Sistema Nacional de Avaliação da Educação Superior (Sinaes), instituído pela Lei $\mathrm{n}^{\circ} 10.861$, de 14 de abril de 2004 (BRASIL, 2017). O Sinaes avalia a educação superior por meio de vários instrumentos, que focam na instituição, no curso e no estudante. Os processos avaliativos são coordenados e supervisionados pela Comissão Nacional de Avaliação da Educação Superior (Conaes), que organiza e coordena externamente o processo avaliativo. A operacionalização é de responsabilidade do Instituto Nacional de Estudos e Pesquisas Educacionais Anísio Teixeira (Inep), que desenvolve as práticas avaliativas que lhe forem designadas (FONSECA; NAMEN, 2016). As informações obtidas com o Sinaes são utilizadas pelos vários segmentos da comunidade, incluindo as Instituições de Ensino Superior (IES), os órgãos governamentais, os alunos e o público em geral, de maneira a orientar suas decisões quanto à realidade dos cursos e das instituições.

Para a avaliação dos estudantes foi implementado o Exame Nacional de Desempenho de Estudantes (Enade) (BRASIL, 2016a), um exame obrigatório que é aplicado periodicamente, em ciclos de três anos, aos alunos de todos os cursos de graduação. Além de avaliar e acompanhar o processo de aprendizagem e o desempenho acadêmico dos estudantes em relação aos conteúdos programáticos previstos nas diretrizes curriculares do respectivo curso de graduação, o Enade também avalia suas habilidades para ajustar-se às exigências decorrentes da evolução do conhecimento e competências para compreender temas exteriores ao âmbito específico da profissão escolhida, ligados à realidade brasileira e mundial e a outras áreas do conhecimento. Além dos testes de conhecimento, os alunos devem responder a um questionário para levantar a percepção dos alunos sobre o teste e outro sobre o perfil socioeconômico educacional do aluno (SILVA, 2012). 
O Inep aplica também o Exame Nacional do Ensino Médio (Enem), criado em 1998, com o intuito de avaliar a Educação Básica, que apesar de não estar ligado diretamente à avaliação da educação superior, tem grande impacto nesse nível de ensino por ser usado como nota de entrada para um grande número de IES, substituindo completamente, em muitos casos, o Vestibular (BRASIL, 2016b).

Atualmente, o exame é composto de 180 questões, distribuídas em quatro provas objetivas: Ciências Humanas e suas Tecnologias (História, Geografia, Filosofia e Sociologia); Ciências da Natureza e suas Tecnologias (Química, Física e Biologia); Linguagens, Códigos e suas Tecnologias (Língua Portuguesa, Literatura, Língua Estrangeira - Inglês ou Espanhol, Artes, Educação Física e Tecnologias da Informação e Comunicação); Matemática e suas Tecnologias (Matemática). Além disso, os alunos devem fazer uma redação e responder a um questionário socioeconômico-cultural, com preenchimento não obrigatório, a ser respondido no dia da inscrição do exame (SILVA; MORINO; SATO, 2014).

Além de disponibilizar os dados de forma anonimizada, o Inep gera relatórios dessas provas. O Enade gera relatórios de Curso, de IES e Síntese de Área, com estatísticas geradas a partir dos dados. Essas informações incluem o desempenho (mínimo, máximo, média, mediana, desvio-padrão, etc.) dos estudantes na prova, a percepção deles sobre a prova (porcentagem de respostas do questionário de percepção sobre a prova por item), os resultados da Análise do Questionário do Estudante (incluindo a porcentagem de respostas desse questionário por item) e as estatísticas das questões da prova (porcentagem de acertos das questões objetivas e média das notas nas questões discursivas) (BRASIL, 2016a). O Enem disponibiliza as médias e os percentuais de alunos em cada um dos quatro níveis de proficiência e da redação dos estudantes que participaram da prova, por escola, para cada uma das áreas de conhecimento consideradas.

As análises disponibilizadas geralmente se resumem a estatísticas descritivas dos dados, que visam descrever e resumir as informações coletadas. Análises mais sofisticadas, envolvendo estatística inferencial ${ }^{1}$ e/ou mineração de dados ${ }^{2}$, que propõe abstrair informações relevantes a partir de grandes volumes de dados (KULKARNI; RAMPURE; YADAV, 2013), não são realizadas pelo Inep. Nesse contexto, propõe-se esta revisão sistemática, que tem

\footnotetext{
1 A estatística inferencial, ou estatística indutiva, analisa os dados visando aprendizado sobre a população.

2 É uma área do conhecimento que permite estabelecer relações e interpretações que servem de base para a construção de novos conhecimentos. As técnicas de mineração de dados também fornecem capacidade de previsão do resultado de uma observação futura.
} 
como objetivo identificar o uso que vem sendo feito dos dados coletados pelo Instituto, para além das análises feitas por ele próprio.

A pesquisa está estruturada em seis seções. A seção 2 descreve o método de revisão adotado neste trabalho, com foco no planejamento e na condução da RS. A seção 3 apresenta a análise dos resultados obtidos nessa revisão por meio das respostas para as questões de pesquisa. A seção 4 traz uma discussão dos resultados e a seção 5 discorre sobre as considerações, fazendo uma síntese desta pesquisa.

\section{Revisão sistemática da literatura}

Essa revisão sistemática da literatura (RSL) teve como referencial o trabalho de Kitchenham e Charters (2007), que sugere um processo dividido em três estágios: planejamento, composto pela etapa de identificação da necessidade de uma revisão e pela da definição de um protocolo; condução, que compreende a identificação de pesquisas, seleção e avaliação de estudos, extração e síntese dos dados; e reportagem da revisão, que é uma fase única. A ferramenta escolhida para gerenciar esta revisão foi a State of the Art through Systematic Reviews - StArt (UNIVERSIDADE FEDERAL DE SÃO CARLOS, 2013).

Essa RSL foi guiada por uma questão de pesquisa principal: que tipos de análise têm sido realizadas com base nos dados do Enem e do Enade e quais são os seus objetivos?

Este estudo utilizou a string: (enem OR enade) na base de dados Google Scholar (scholar.google.com.br/), uma biblioteca digital consolidada, que possibilita a utilização de strings de busca com palavras-chave e possui acesso online. Foram extraídos artigos de periódicos ou de conferências, dissertações e teses publicados entre 2005 e 2016, escritos em inglês ou português e relevantes para a pesquisa. Foram excluídos aqueles trabalhos que não tratam de Enem e/ou Enade ou que não respondem às questões de pesquisa.

A pesquisa retornou 1.064 trabalhos, distribuídos entre artigos, dissertações e teses. Destes, 1.008 foram excluídos por não tratarem de Enem e/ou de Enade ou por não responderem à questão de pesquisa. Dos 56 trabalhos restantes, foram mantidos 54 após a realização de uma leitura completa. Os dois artigos excluídos na fase de extração aplicavam questionários próprios, relacionados à percepção de docentes sobre os exames. Esses questionários não eram fornecidos pelo Inep e tampouco utilizavam dados extraídos das provas. 
Desse modo, foram selecionados 39 trabalhos que tratam do Enade (Figura 1) e 17 estudos que tratam do Enem (Figura 2), sendo os artigos de Bianchi (2010) e Silveira (2013) contabilizados em dobro por tratarem tanto do Enade quanto do Enem.

Figura 1 - Trabalhos sobre Enade agrupados por ano e natureza.

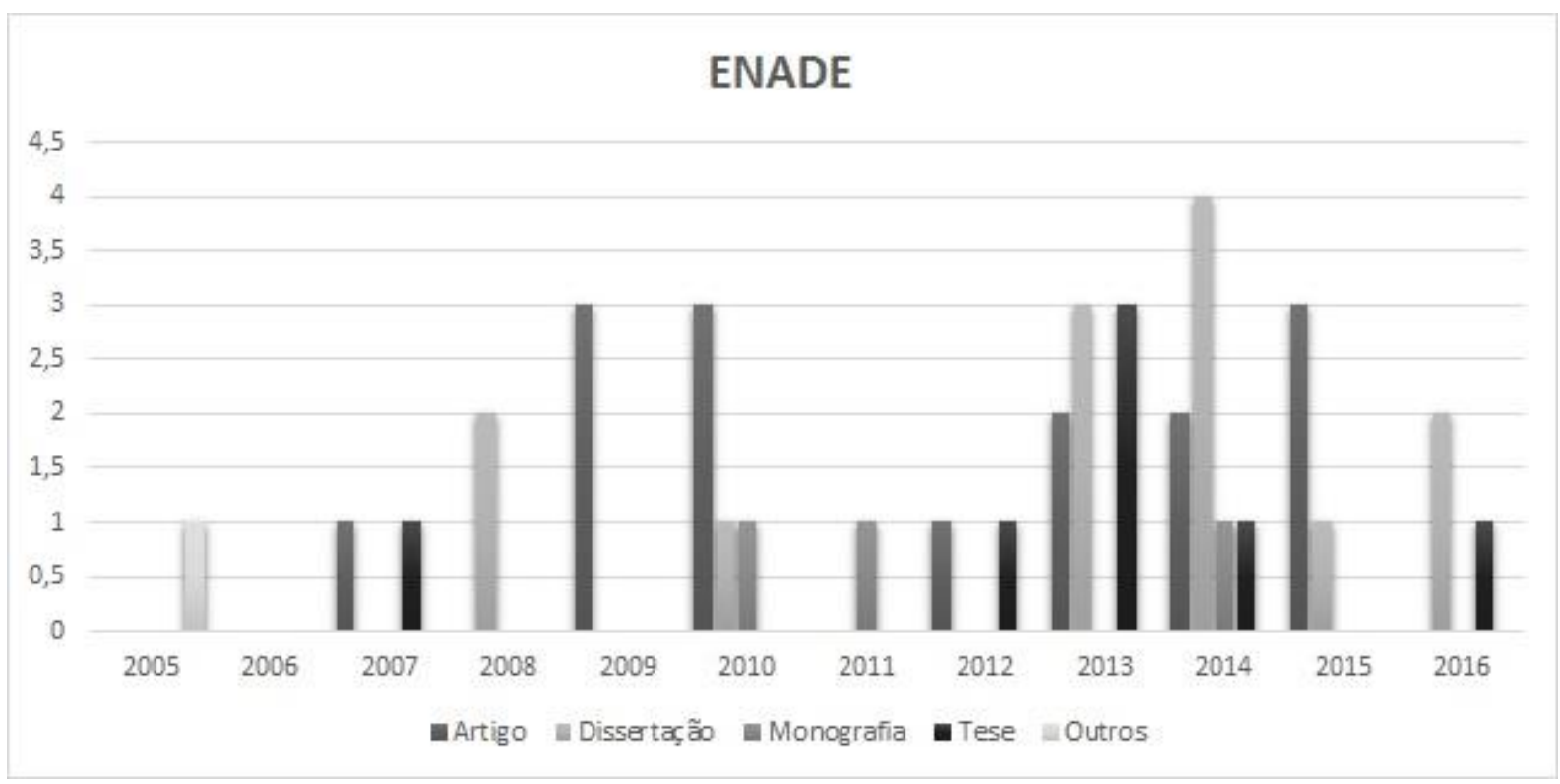

Figura 2 - Trabalhos sobre o Enem agrupados por ano e natureza.

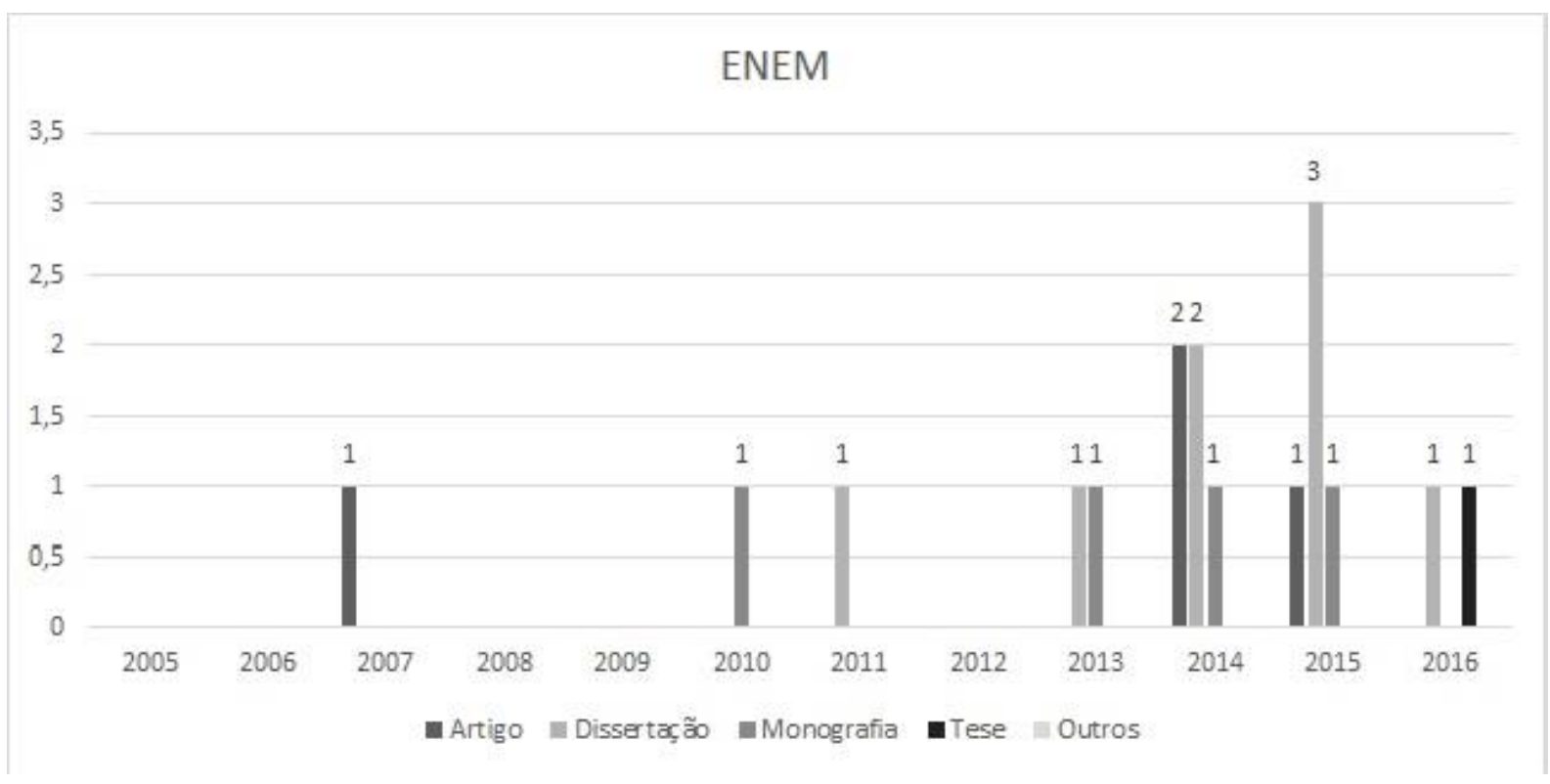

É possível observar que as análises relacionadas ao Enem e ao Enade se concentram em maior volume nos últimos anos, de 2013 a 2016, apesar dos exames serem realizados 
desde 1998 (Enem) e 2005 (Enade). O interesse pelos resultados do Enem pode estar associado ao seu uso como forma de acesso ao Ensino Superior no Brasil, enquanto o Enade tem se consolidado e adquirido um formato mais estável nos últimos anos.

\section{Resultados e Discussão}

Para responder à questão de pesquisa - quais tipos de análise têm sido realizados com base nos dados do Enem e do Enade e quais são os seus objetivos? - ela foi desdobrada em duas partes: quais os objetivos das análises e os problemas que as motivaram? E que tipo de análise foi feita?

Os objetivos encontrados foram organizados em seis categorias, conforme as Tabelas 1 (Enem) e 2 (Enade).

Tabela 1 - Relação de trabalhos e os objetivos de suas análises - Enem

\begin{tabular}{|c|c|c|}
\hline Objetivo da Análise & Referências & Qtd \\
\hline Conteúdo/Conhecimento & $\begin{array}{l}\text { Bianchi (2010), França (2016), Oliveira (2014), Ferreira, Edson } \\
\text { Martins (2014). }\end{array}$ & 4 \\
\hline Administrativo & Guerra, Nakamura e Hruschka (2014), Lima (2011), Parente (2014). & 3 \\
\hline Desempenho/Rendimento & $\begin{array}{l}\text { Gonçalves, 2015, Moreira (2015), Oliveira (2013), Silva, S. M. } \\
\text { (2015), Silva e Meletti (2012), Silva, Morino e Sato (2014), Silveira } \\
\text { (2013), Soares (2014). }\end{array}$ & 8 \\
\hline Testar/desenvolver ferramentas & Costa (2016), Silva, A. L. (2015). & 2 \\
\hline Total & ------- & 17 \\
\hline
\end{tabular}

Fonte: Elaboração própria.

Tabela 2 - Relação de Trabalhos e os objetivos de suas análises - ENADE

\begin{tabular}{|c|c|c|}
\hline Objetivo da Análise & Referências & Qtd \\
\hline Conhecimento/ Conteúdo & $\begin{array}{l}\text { Bianchi (2010), Lopes e Vendramini (2015), Primi et al. (2010), Brito } \\
\text { (2015). }\end{array}$ & 4 \\
\hline Administrativo & $\begin{array}{l}\text { Gomes (2011), Lavor, Andriola e Lima (2016), Rossetto (2016), Silveira } \\
\text { (2013). }\end{array}$ & 4 \\
\hline Desempenho/Rendimento & $\begin{array}{l}\text { Almeida (2014), Barreyro e Aureliano (2009), Batista (2014), Brito (2007), } \\
\text { Costa e Oliveira (2013), Dal-Farra et al. (2012), Dias, Porto e Nunes (2016), } \\
\text { Gaudio (2014), Gontijo (2014), Guerra } \text { et al. (2010), Moriconi e Nascimento } \\
\text { (2014), Neto Santos (2016), Novossate (2010), Silva (2012), Silva (2016), } \\
\text { Silva, Vendramini e Lopes (2010), Souza (2008), Verhine e Dantas (2009), } \\
\text { Waltenberg e Carvalho (2013). }\end{array}$ & 19 \\
\hline $\begin{array}{l}\text { Testar/Desenvolver } \\
\text { Ferramentas }\end{array}$ & $\begin{array}{l}\text { Álvares, Campos e Gomes (2015), Corrêa (2013), Gotti (2013), Machado } \\
\text { (2008), Pires (2013). }\end{array}$ & 5 \\
\hline $\begin{array}{l}\text { Avaliação/Estrutura do } \\
\text { Exame }\end{array}$ & Costa e Martins (2014), Verhine e Dantas (2005), Rodrigues (2013). & 3 \\
\hline $\begin{array}{l}\text { Formação/Qualificação } \\
\text { Docente }\end{array}$ & $\begin{array}{l}\text { Brito (2015), Ferreira, Marcos Felipe (2014), Lara (2007), Oliveira (2014), } \\
\text { Schwengber (2013). }\end{array}$ & 5 \\
\hline Total & ----------------- & 40 \\
\hline
\end{tabular}

Fonte: Elaboração própria. 
Trabalhos classificados na categoria 'Conhecimento/Conteúdo' têm como objetivo fazer um estudo sobre o conteúdo das provas e/ou o conhecimento dos estudantes em determinados assuntos abordados nos exames. Lopes e Vendramini (2015), por exemplo, avaliam as propriedades psicométricas da prova de Pedagogia no Enade e Bianchi (2010) analisa o percentual de questões envolvendo a interpretação de informações apresentada em gráficos e tabelas estatísticas no Enade e Enem.

As investigações feitas com o objetivo de análise 'Administrativo' abrangem aspectos de gestão, como o acesso ao Ensino Superior (GUERRA; NAKAMURA; HRUSCHKA, 2014; PARENTE, 2014) e o impacto que os resultados do Enade têm sobre as questões acadêmicas, como mudanças no currículo e na cultura institucional (GOMES, 2011).

As análises que objetivam o 'Desempenho/Rendimento' avaliam os resultados dos estudantes e/ou das instituições nos exames. Dentre essas análises destaca-se a de Souza (2008), que busca identificar a relação entre o desempenho dos alunos e a sua situação socioeconômica. Outros trabalhos focam o 'Desenvolvimento e Teste de ferramentas', com o propósito de auxiliar na análise dos resultados dos exames, como o estudo de Costa (2016), com a ferramenta Vis-Scholar, que utiliza estatística para correlacionar conjuntos de dados e métodos de visualização dessas correlações, a fim de facilitar a visualização dos dados.

'Avaliação/Estrutura do exame' refere-se a trabalhos que procuram entender de que forma o exame é estruturado e até fazem uma avaliação comparando com outros exames, como, por exemplo, o Provão (VERHINE; DANTAS, 2006). 'Formação/Qualificação Docente' agrupa trabalhos onde os dados dos exames são analisados a fim de aprimorar a formação do docente e relacioná-la com o desempenho dos estudantes nas provas. Nessa categoria encontra-se o trabalho de Brito (2015), que objetiva determinar como as características do corpo docente de uma IES influenciam o desempenho dos concluintes no Enade.

As análises mais recorrentes nos trabalhos relacionadas são feitas com o objetivo de estudar as condições de acesso e permanência no ensino superior e também há um número expressivo para os trabalhos que analisaram os dados do Enade e Enem com o objetivo de verificar o desempenho e traçar os perfis dos estudantes. É importante salientar que alguns trabalhos, como o de Brito (2015), possuíam dois objetivos de análise: conteúdo/conhecimento e formação/qualificação docente, portanto foram contabilizados em dobro. 
Os objetivos apresentados surgiram a partir de problemas detectados pelos pesquisadores e que motivaram os trabalhos realizados, visando contribuir para a solução. As Tabelas 3 (Enem) e 4 (Enade) relacionam os problemas que os pesquisadores tentaram resolver.

Tabela 3 - Relação de trabalhos e os problemas abordados - Enem

\begin{tabular}{|c|c|c|}
\hline Problema & Referências & Qtd \\
\hline $\begin{array}{l}\text { Dificuldade na } \\
\text { avaliação e análise } \\
\text { de dados }\end{array}$ & Costa (2016). & 1 \\
\hline Qualidade do Ensino & $\begin{array}{l}\text { Bianchi (2010), França (2016), Oliveira (2013), Santos e Concordino (2015), } \\
\text { Silva, Morino e Sato (2014), Silveira (2013), Soares (2014), Silva, A. L. (2015), } \\
\text { Silva, S. M. (2015). }\end{array}$ & 9 \\
\hline Desempenho & $\begin{array}{l}\text { Ferreira, Edson Martins (2014), Gonçalves (2015), Lima (2011), Moreira (2015), } \\
\text { Oliveira (2013), Silva e Meletti (2012). }\end{array}$ & 6 \\
\hline Administrativo & Guerra, Nakamura e Hruschka (2014), Parente (2014). & 2 \\
\hline Total & 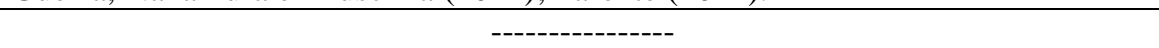 & 18 \\
\hline
\end{tabular}

Fonte: Elaboração própria.

Tabela 4 - Relação de Trabalhos e os problemas abordados - Enade

\begin{tabular}{|c|c|c|}
\hline Problema & Referências & Qtd \\
\hline $\begin{array}{l}\text { Dificuldade na } \\
\text { visualização e análise de } \\
\text { dados }\end{array}$ & Álvares, Campos e Gomes (2015), Gotti (2013). & 2 \\
\hline Qualidade do Ensino & $\begin{array}{l}\text { Gontijo (2014), Guerra et al. (2010), Lavor, Andriola e Lima (2016), Lopes e } \\
\text { Vendramini (2015), Machado (2008), Neto Santos (2016), Pires (2013), Primi } \\
\text { et al., (2010), Rodrigues (2013), Silva (2012), Verhine e Dantas (2006, 2009), } \\
\text { Silveira (2013). }\end{array}$ & 13 \\
\hline Desempenho & $\begin{array}{l}\text { Almeida (2014), Barreyro e Aureliano (2009), Bianchi (2010), Brito (2007), } \\
\text { Brito (2015), Corrêa (2013), Costa e Oliveira (2013), Dias, Porto e Nunes } \\
\text { (2016), Gaudio (2014), Lavor, Andriola e Lima (2016), Moriconi e } \\
\text { Nascimento (2014), Silva (2016), Souza (2008), Waltenberg e Carvalho } \\
\text { (2013). }\end{array}$ & 14 \\
\hline Administrativo & Batista (2014), Gomes (2011), Rosseto (2016). & 3 \\
\hline $\begin{array}{l}\text { Formação/Qualificação } \\
\text { Docente }\end{array}$ & $\begin{array}{l}\text { Costa e Martins (2014), Dal-Farra et al. (2012), Ferreira, Marcos Felipe } \\
\text { (2014), Lara (2007), Novossate (2010), Oliveira (2014), Schwengber (2013). }\end{array}$ & 7 \\
\hline Total & ------------------ & 39 \\
\hline
\end{tabular}

Fonte: Elaboração própria.

Problemas relacionados à 'Dificuldade na visualização e análise de dados' motivaram pesquisas que visam facilitar as análises dos dados. Isso inclui o desenvolvimento de ferramentas, como é o caso do estudo de Álvares, Campos e Gomes (2015). Dificuldades relacionadas à 'Qualidade do Ensino' instigam os pesquisadores a encontrarem métodos que visam melhorar o processo de ensino e, consequentemente, a contribuírem para o aumento da qualidade da educação. Problemas de 'Desempenho', abordam estudos que procuram verificar como estudantes de diferentes perfis se desenvolvem na academia. Gaudio (2014) destaca-se 
por investigar a inclusão social do estudante bolsista do Programa Universidade para Todos (ProUni). Dificuldades 'Administrativas' incluem questões como evasão, número de vagas, mudanças no currículo dos cursos e na cultura da instituição. Já os problemas de 'Formação/Qualificação Docente' incluem questões como a melhoria do trabalho dos docentes a partir do conhecimento das provas e de como trabalhar os estudantes que as farão.

$\mathrm{Na}$ busca das soluções para os referidos problemas, os trabalhos utilizam diferentes tipos de análises sobre os microdados gerados a partir dos exames. É importante salientar que alguns trabalhos analisaram mais que um tipo de dado dos exames, estes foram contabilizados de acordo com a diversificação de dados utilizados. Os tipos de análises e os dados utilizados estão apresentados nas Tabelas 5 (Enem) e 6 (Enade).

Tabela 5 - Relação do tipo de análise e dados abordados - Enem

\begin{tabular}{|c|c|c|c|}
\hline Análise & Dados usados & Referências & Qtd \\
\hline Estatística & Notas do ENEM & $\begin{array}{l}\text { Costa (2016), Gonçalves (2015), Lima (2011), Moreira (2015), } \\
\text { Oliveira (2013), Parente (2014), Silva, S. M. (2015), Silveira } \\
\text { (2013). }\end{array}$ & 8 \\
\hline Estatística & Questões da Prova & $\begin{array}{l}\text { Bianchi (2010), Ferreira, Edson Martins (2014), França (2016), } \\
\text { Santos e Concordino (2015), Silva, A. L. (2015). }\end{array}$ & 5 \\
\hline Estatística & $\begin{array}{l}\text { Número de Alunos } \\
\text { Inscritos no ENEM } \\
\text { por ano }\end{array}$ & Guerra, Nakamura e Hruschka (2014). & 1 \\
\hline Estatística & Leitura dos Relatórios & Soares (2014). & 1 \\
\hline Estatística & $\begin{array}{l}\text { Questionário } \\
\text { Socioeconômico }\end{array}$ & Gonçalves (2015), Parente (2014), Silva e Meletti (2012). & 3 \\
\hline $\begin{array}{l}\text { Mineração } \\
\text { de Dados }\end{array}$ & Notas do ENEM & Silva, Morino e Sato (2014). & 1 \\
\hline Total & -------- & -------------- & 19 \\
\hline
\end{tabular}

Fonte: Elaboração própria.

Tabela 6 - Relação do tipo de análise e dados abordados - Enade

\begin{tabular}{|c|c|c|c|}
\hline Análise & Dados usados & Referências & Qtd \\
\hline Estatística & Notas do ENADE & $\begin{array}{l}\text { Almeida (2014), Brito (2007), Brito (2015), Dal-Farra et al. (2012), } \\
\text { Dias, Porto e Nunes (2016), Gaudio (2014), Gontijo (2014), } \\
\text { Moriconi e Nascimento (2014), Oliveira (2014), Rossetto (2016), } \\
\text { Silva (2016), Silva, Vendramini e Lopes (2010), Souza (2008), } \\
\text { Verhine e Dantas (2006, 2009), Waltenberg e Carvalho (2013). }\end{array}$ & 16 \\
\hline Estatística & $\begin{array}{c}\text { Questionário } \\
\text { Socioeconômico }\end{array}$ & $\begin{array}{l}\text { Almeida (2014), Barreyro e Aureliano (2009), Brito (2007), Ferreira, } \\
\text { Marcos Felipe (2014), Gontijo (2014), Lavor, Andriola e Lima } \\
\text { (2016), Moriconi e Nascimento (2014), Rossetto (2016), Souza } \\
\text { (2008), Verhine e Dantas (2009). }\end{array}$ & 10 \\
\hline Estatística & Conteúdo de Prova & $\begin{array}{l}\text { Bianchi (2010), Costa e Martins (2014), Lara (2007), Lopes e } \\
\text { Vendramini (2015), Novossate (2010), Pires (2013), Primi et al. } \\
\text { (2010), Schwengber (2013). }\end{array}$ & 8 \\
\hline Estatística & $\begin{array}{l}\text { Conceito do } \\
\text { ENADE }\end{array}$ & $\begin{array}{l}\text { Batista (2014), Corrêa (2013), Costa (2016), Costa e Oliveira (2013), } \\
\text { Gotti (2013), Guerra et al. (2010), Machado (2008), Silva (2012). }\end{array}$ & 8 \\
\hline Estatística & $\begin{array}{l}\text { Questionário do } \\
\text { Coordenador }\end{array}$ & Gomes (2011). & 1 \\
\hline
\end{tabular}




\begin{tabular}{|c|c|c|c|}
\hline Estatística & $\begin{array}{l}\text { Questionário de } \\
\text { Percepção do } \\
\text { Estudante }\end{array}$ & $\begin{array}{l}\text { Gaudio (2014), Gontijo (2014), Neto Santos (2016), Rodrigues } \\
\text { (2013). }\end{array}$ & 4 \\
\hline $\begin{array}{l}\text { Mineração } \\
\text { de Dados }\end{array}$ & Notas do ENADE & Álvares, Campos e Gomes (2015). & 1 \\
\hline Total & --------- & ------- & 48 \\
\hline
\end{tabular}

A estatística é a principal ferramenta de análise, com variações nos testes aplicados e nas abordagens. Alguns trabalhos utilizaram regressão linear - como os de Guerra, Nakamura e Hruschka (2014), Oliveira (2014), Silva (2016), Batista (2014) e Corrêa (2013) -, outros a fatorial - como Batista (2014), Pires (2013) e Santos Neto (2016) -, mas a maioria utilizou a Estatística Descritiva. Os principais softwares usados foram:

- Statistical Package for the Social Sciences (SPSS), um software de apoio à tomada de decisão que inclui aplicação analítica, Data Mining, Text Mining e estatística, que transformam os dados em informações importantes;

- Matrix Laboratory (Matlab), que é um software interativo que integra análise numérica, cálculo em matrizes, processamento de sinais e construção de gráficos;

- Data Analysis and Statistical Software (STATA), um software de estatística usado para análise econométrica, utilizando dados cross-section, dados em painel e estimação de séries temporais. Também tem recursos potentes de tabulação de variáveis e comandos para cálculo das medidas de associação usadas em epidemiologia, como razão de incidências, risco relativo, razão de chances e risco atribuível.

A Mineração de Dados foi utilizada em dois (02) dos artigos selecionados. A fim de diminuir a complexidade de análise dos dados divulgados pelo Inep (ALVARES; CAMPOS; GOMES, 2015), aplicam a mineração de dados nos resultados do Enade. O objetivo foi desenvolver um aplicativo para auxiliar os usuários a obterem informações úteis e íntegras a respeito de qualquer curso participante do exame. A ferramenta utilizou o sistema gerenciador de banco de dados MySQL e o QlikView 11 Desktop, para fazer análises comparativas e descritivas dos resultados do Enade nos anos de 2007 e 2010.

Silva, Morino e Sato (2014) investigam os motivos que influenciam no desempenho dos alunos no Enem. Para isso, mineraram as notas das provas e os questionários socioeconômicos dos estudantes e conseguiram extrair informações importantes, como as de que a renda familiar baixa, a escolaridade dos pais de nível primário e a quantidade alta de pessoas que moram com os estudantes são atributos que diminuem o desempenho do aluno. A análise é feita por associação de dados usando o algoritmo A Priori. As ferramentas usadas 
neste trabalho foram o Oracle Express Edition $11 \mathrm{~g}$ e o PL/SQL Developer, dois softwares que permitem a extração dos dados para a pesquisa. Para os experimentos, foi utilizada a ferramenta RapidMiner 5.1.

As conclusões dos trabalhos selecionados comprovam as teorias estabelecidas, como a de que quanto mais se investe em educação, melhores são os resultados (COSTA, 2016) e a de que além dos investimentos é preciso manter uma avaliação constante do sistema educacional (RODRIGUES, 2013; VERHINE; DANTAS, 2009). Por sua vez, Santos e Concordido (2015) sugerem que o Enem não deveria ser o regulador do Ensino Médio, portanto, não deveria definir o que prevalece ou não nos currículos.

Os artigos também reafirmam a importância da qualificação contínua dos professores. Oliveira (2014) destaca a influência de características como formação e título de especialização dos docentes nos resultados do Enade, enquanto Dal-Farra et al. (2012) o regime de dedicação do corpo docente.

No contexto social, os resultados sugerem que as diversas políticas de ações afirmativas foram bem-sucedidas no objetivo de proporcionar mais diversidade nas universidades (WALTENBERG; CARVALHO, 2013). Por outro lado, Silva e Meletti (2012) constatam que, apesar da implementação das políticas inclusivas, a participação dos alunos com necessidades educacionais especiais é mínima, o que denota a ideia de ambiguidade entre discurso e prática inclusiva.

\section{Considerações}

Essa revisão apresenta os tipos de análises e seus objetivos, tendo sido realizada nas bases de dados do Enem e do Enade. Analisaram-se os problemas abordados pelos trabalhos encontrados e as ferramentas usadas. A fim de seguir uma pesquisa sistemática, apenas as publicações que se encaixavam em todos os critérios de inclusão foram selecionadas.

A leitura dos trabalhos selecionados mostrou que as análises sobre o Enem/Enade, em sua maioria, são feitas com o objetivo de melhorar o desempenho dos estudantes nos exames. Os problemas que impulsionam essas análises estão ligados à qualidade da educação.

A quantidade de estudos feitos sobre o Enade é expressivamente maior que sobre o Enem e poucos tentam analisar os dois. Isso pode estar relacionado ao fato de que frequentemente se deseja analisar o desempenho dos alunos e os dados do Enade podem ser analisados por curso. Vale observar que essas análises são geralmente feitas por pesquisadores usando os dados associados aos cursos de seu interesse e para os quais desejam contribuir. 
Observou-se também a diversidade nas áreas pesquisadas. Foram explorados diversos níveis de tomada de decisão: aluno, docente, curso, instituição, governo e sociedade. Para a realização dos estudos, foram analisados diferentes tipos de dados e os mais utilizados foram as notas dos exames e os questionários socioeconômicos. Os conteúdos das provas foram analisados apenas em estudos que abordavam o Enade e as análises foram direcionadas para interpretações do conteúdo, sem que nenhuma categorização fosse realizada.

O tipo de análise mais utilizada nas bases de dados do Enem e do Enade é a estatística descritiva, mostrando a necessidade de se investir em pesquisas que abordem outros tipos de análises, como a mineração de dados. Apenas dois artigos a utilizaram e ela foi aplicada nas notas dos exames. Com o vasto volume de dados fornecidos por ambos os exames, esse tipo de análise pode ter uma amplitude maior, permitindo análises de perfil ou de predição de resultados, que correlacionam dados socioeconômicos dos alunos, notas da prova (inclusive permitindo a análise do conteúdo das provas) e informações sobre a estrutura das instituições, dados pouco explorados pelos trabalhos analisados.

Além disso, análises inferenciais permitem verificar se é possível generalizar os resultados obtidos na amostra para a população em geral, permitindo verificar correlação significativa entre diferentes grupos, tais como sexo (feminino, masculino), regiões do Brasil (Norte, Nordeste, Sul, Sudeste, Centro-Oeste), tipo de instituição (pública, privada) etc., ou verificar a correlação em diferentes momentos temporais, propiciando estudos longitudinais, que analisam o comportamento dos alunos em diferentes momentos ou situações. Infelizmente, esse tipo de análise fica limitada pelo fato de não ser possível associar os dados de um mesmo aluno nas diversas provas realizadas pelo Inep, o que permitiria analisar o percurso dos alunos. Caso fosse possível integrar as bases do Enem e do Enade, poderia se analisar o aluno, da entrada à graduação no Ensino Superior.

Existe, portanto, um vasto campo de pesquisa ainda inexplorado e com potencial para gerar informação útil para compreender melhor o perfil dos alunos e o sistema educacional brasileiro. Além disso, pode-se fazer predições a respeito do desempenho acadêmico dos alunos ou de outros aspectos relevantes, o que permite a definição de ações preventivas que visem evitar esses problemas. 


\section{Referências}

ALMEIDA, Álvaro Cavalcanti de. Modelo de mensuração do desempenho dos institutos federais: uma análise a partir de microdados. Dissertação (Mestrado Profissional em Gestão em Organizações Aprendentes) - Universidade Federal da Paraíba, João Pessoa, 2014. Disponível em: https://repositorio.ufpb.br/jspui/bitstream/tede/5913/1/arquivototal.pdf. Acesso em: 15 maio 2017.

ALVARES, Reinaldo Viana; CAMPOS, Nathielly de Souza; GOMES, Vinicius Benter. Adoção de Data Discovery para apoio ao processo de análise de dados do Enade. In: CONGRESSO INTERNACIONAL DE INFORMÁTICA EDUCATIVA, 20., 2015, Santiago. Anais[...]. Santiago: Universidade de Brasília, 2015. Disponível em: http://www.tise.cl/volumen11/TISE2015/480-485.pdf. Acesso em: 02 abr. 2017.

BARREYRO, Gladys Beatriz; AURELIANO, Arlei Flausino. Características dos ingressantes de universidades públicas do estado de São Paulo: novos campi, velhas desigualdades?

Educere et Educare - Revista de Educação, Cascavel, PR, v. 2, n. 10, p. 1-25, 2009. Disponível em: http: //e-

revista.unioeste.br/index.php/educereeteducare/article/view/5090/4027. Acesso em: 2 abr. 2017

BATISTA, Lídia Melo. Estratégias acadêmicas na avaliação de desempenho dos Cursos de Ciências Contábeis no Enade em Natal - RN. 2014. Dissertação (Mestrado) Universidade Federal do Rio Grande do Norte, Natal, 2014. Disponível em: http://www.repositorio.ufrn.br:8080/jspui/bitstream/123456789/15091/1/LidiaMB_DISSERT. pdf. Acesso em: 02 abr. 2017.

BIANCHI, Juliane Mucio. Leitura e interpretação de tabelas e gráficos pelos alunos do Curso de Matemática da URI - Campus de Erechim. 2010. Monografia (Graduação em Matemática) - Universidade Regional do Alto Uruguai e das Missões, Erechim, 2010. Disponível em: http://www.uricer.edu.br/cursos/arq_trabalhos_usuario/1267.pdf. Acesso em: 10 maio 2017.

BRASIL. Instituto Nacional de Estudos e Pesquisas Educacionais Anísio Teixeira. Enade. Brasília, 2016a. Disponível em: http://portal.inep.gov.br/enade. Acesso em: 18 dez. 2016.

BRASIL. Instituto Nacional de Estudos e Pesquisas Educacionais Anísio Teixeira. Sinaes. Brasília, 2017. Disponível em: http://portal.inep.gov.br/web/guest/sinaes. Acesso em: 6 maio. 2017.

BRASIL. Instituto Nacional de Estudos e Pesquisas Educacionais Anísio Teixeira. Sobre o Enem - Inep. Brasília, 2016b. Disponível em: http://portal.inep.gov.br/web/enem/sobre-oenem. Acesso em: 18 dez. 2016.

BRITO, Márcia Regina F. de. Enade 2005: perfil, desempenho e razão da opção dos estudantes pelas Licenciaturas. Avaliação, Campinas; Sorocaba, v. 12, n. 3, p. 401-443, 2007. Disponível em: http://www.scielo.br/scielo.php?pid=S141440772007000300003\&script=sci_abstract\&tlng=pt. Acesso em: 18 dez. 2016.

BRITO, Tainá Fernandes. Corpo docente: fatores determinantes do desempenho discente no Enade. 2015. Dissertação (Mestrado em Administração) - Universidade de São Paulo, São Paulo, 2015. Disponível em: http://www.teses.usp.br/teses/disponiveis/12/12139/tde21032016-115045/en.php. Acesso em: 31 jan 2017.

CORREAA, Denise Maria Moreira Chagas. Indicadores de gestão do TCU e o desempenho dos cursos no Enade: um estudo de cursos de graduação. 2013. Tese (Doutorado em 
Educação Brasileira) - Universidade Federal do Ceará, Fortaleza, 2013. Disponível em: http://www.repositorio.ufc.br/bitstream/riufc/5943/3/2013_tese_dmmccorrea.pdf. Acesso em: 26 mar. 2017.

COSTA, Jean Carlos Araújo. Vis-scholar: uma metodologia de visualização e análise de dados na Educação. 2016. Dissertação (Mestrado em Computação Aplicada) - Universidade do Vale do Rio dos Sinos, São Leopoldo, 2016. Disponível em:

http://www.repositorio.jesuita.org.br/handle/UNISINOS/5280. Acesso em: 26 mar. 2017.

COSTA, João Paulo de Castro; MARTINS, Maria Inês. O ENADE para a licenciatura em física: Uma proposta de matriz de referência. Revista Brasileira de Ensino de Física, São Paulo, SP, v. 36, n. 3, p. 3401-3419, 2014. Disponível em:

http://www.sbfisica.org.br/rbef/pdf/363401.pdf. Acesso em: 27 de nov. 2016.

COSTA, Marcos Vinícius de Oliveira; OLIVEIRA, Vanderli Fava de. A evolução do desempenho das engenharias no Enade. In: CONGRESSO BRASILEIRO DE EDUCAÇÃO EM ENGENHARIA, 41., 2013, Gramado, RS. Anais [...]. Gramado, RS: Associação Brasileira de Educação em Engenharia, 2013. Disponível em: http://www.ufjf.br/observatorioengenharia/files/2012/01/enegep2013_TN_STO_177_007_23 248.pdf. Acesso em: 3 dez. 2016.

DAL-FARRA, Rossano André et al. Cursos de graduação em Ciências: uma análise dos dados do Exame Nacional de Desempenho dos Estudantes - Enade. Revista Dynamis, Blumenau, SC, v. 18, n. 1, p. 3-20, 2012. Disponível em:

http://proxy.furb.br/ojs/index.php/dynamis/article/view/3217. Acesso em: 15 abr. 2017.

DIAS, Juliana da Silva; PORTO, Cristiane de Magalhães; NUNES, Andrea Karla Ferreira. Formação geral e conhecimento específico na prova Enade. In: ENCONTRO

INTERNACIONAL DE FORMAÇÃO DE PROFESSORES, 9., 2016, Sergipe. Anais [...]. Sergipe: Instituto de Tecnologia e Pesquisa, 2016. Disponível em: https://eventos.set.edu.br/index.php/enfope/article/view/2056. Acesso em: 12 jan. 2017.

FERREIRA, Edson Martins. Análise da abrangência da matriz de referência do Enem com relação às habilidades avaliadas nos itens de Matemática aplicados de 2009 a 2013. 2014. Dissertação (Mestrado Profissional em Matemática em Rede Nacional) - Universidade de Brasília, Brasília, 2014. Disponível em: http://repositorio.unb.br/handle/10482/17321. Acesso em: 29 nov. 2016.

FERREIRA, Marcos Felipe. O curso de Pedagogia: perfil de ingresso, inserção profissional e promoção social. 2014. Dissertação (Mestrado em Educação) - Universidade de Brasília, Brasília, 2014. Disponível em: http://repositorio.unb.br/handle/10482/17125. Acesso em: 6 dez. 2016.

FERREIRA, Rosilda Arruda; TENÓRIO, Robinson Moreira. A construção de indicadores de qualidade no campo da avaliação educacional: um enfoque epistemológico. Revista

Lusófona de Educação, Lisboa, n. 15, p. 71-97, 2010. Disponível em: http://www.scielo.mec.pt/pdf/rle/n15/n15a06.pdf. Acesso em: 06 maio 2017.

FONSECA, Stella Oggioni da; NAMEN, Anderson Amendoeira. Mineração em bases de dados do Inep: uma análise exploratória para nortear melhorias no sistema educacional brasileiro. Educação em Revista, Belo Horizonte, v. 32, n. 1, p. 133-157, mar. 2016. Disponível em: http://submission.scielo.br/index.php/edur/article/view/140742. Acesso em: 09 jun 2016.

FRANÇA, Daniela Hilda de Souza Siqueira. A interdisciplinaridade e a contextualização em uma abordagem complexa: análise das provas do Enem com base nos gêneros do 
discurso. 2016. Dissertação (Mestrado em Educação) - Universidade Federal de Goiás, Goiânia, 2016. Disponível em: https://repositorio.bc.ufg.br/tede/handle/tede/5790. Acesso em: 27 nov. 2016.

GAUDIO, Ana Paula de Siqueira. O Prouni como política de inclusão social: uma avaliação por meio do Enade. 2014. Dissertação (Mestrado em Educação) - Universidade Católica de Brasília, Brasília, 2014. Disponível em: https://bdtd.ucb.br:8443/jspui/handle/123456789/783. Acesso em: 30 nov. 2016.

GOMES, Pedro Henrique de Farias. Efeitos do Exame Nacional de Desempenho dos Estudantes/Enade (2008) nas licenciaturas de História e Letras da Universidade de Brasília. 2011. Monografia (Graduação em Pedagogia) - Universidade de Brasília, Brasília, 2011. Disponível em: http://bdm.unb.br/handle/10483/3195. Acesso em: 28 nov. 2016.

GONÇALVES, Fernando. Sucesso no campo escolar de estudantes oriundos de classes populares: estrutura e trajetórias. 2015. Dissertação (Mestrado Sociologia) - Universidade Federal do Rio Grande do Sul, 2015. Disponível em: https://www.lume.ufrgs.br/handle/10183/116492. Acesso em: 06 dez. 2016.

GONTIJO, Simone Braz Ferreira. Implicações do Enade para a organização do trabalho pedagógico e as práticas avaliativas em um curso de Pedagogia. 2014. Tese (Doutorado em Educação) - Universidade de Brasília, Brasília, 2014. Disponível em: http://repositorio.unb.br/handle/10482/16027. Acesso em: 29 nov. 2016.

GOTTI, Fernando José Alho. Proposta de um modelo de avaliação de desempenho de alunos de uma IES utilizando a inteligência computacional. 2013. Tese (Doutorado em Engenharia de Produção) - Universidade Paulista, São Paulo, 2013. Disponível em: https://200.196.224.129/presencial/ensino/pos_graduacao/strictosensu/eng_producao/downloa d/eng_fernandojosealhogotti.pdf. Acesso em: 30 nov. 2016.

GUERRA, Gabriela Ludwig et al. Instituições de ensino superior comunitárias e o Enade. In: SALÃO DE INICIAÇÃO CIENTÍFICA - PUCRS, 11., 2010, Porto Alegre. Anais [...]. Porto Alegre: Pontifícia Universidade Católica do Rio Grande do Sul, 2010. Disponível em: http://www.pucrs.br/edipucrs/XISalaoIC/Ciencias_Humanas/Educacao/83250GABRIELALUDWIGGUERRA.pdf. Acesso em: 28 nov. 2016.

GUERRA, Pedro Calais; NAKAMURA, Rodrigo Yuji Mizobe; HRUSCHKA, Eduardo Raul. Estimativa de demanda potencial de matrículas em ensino superior usando dados públicos e múltiplos modelos de regressão. In: SYMPOSIUM ON KNOWLEDGE DISCOVERY, MINING AND LEARNING, 2., 2014, São Carlos, SP. Proceedings [...]. São Carlos, SP: Sociedade Brasileira de Computação, 2014. Disponível em: http://www2.dc.ufscar.br/ marcela/anaisKDMiLe2014/artigos/SHORT/kdmile2014_Short2.p df. Acesso em: 28 nov. 2016.

KITCHENHAM, Barbara; CHARTERS, Stuart. Guidelines for performing systematic literature reviews in software engineering. 2007. Durham: Department of Computer Science, 2007. Disponível em:

http://citeseerx.ist.psu.edu/viewdoc/download;jsessionid=6EF8E10FF84FC323AB27AE7AF7 D8D1EF?doi=10.1.1.117.471\&rep=rep1\&type=pdf. Acesso em: 06 mar. 2017.

KULKARNI, Suhas G.; RAMPURE, Ganesh C.; YADAV, Bhagwat. Understanding educational data mining. International Journal of Electronics and Computer Science Engineering, Índia, v. 2, n. 2, p. 773-77, 2013. Disponível em:

http://citeseerx.ist.psu.edu/viewdoc/download?doi=10.1.1.294.7653\&rep=rep1\&type=pdf. Acesso em: 14 jun. 2016. 
LARA, Isabel Cristina Machado de. Exames nacionais e as "verdades" sobre a produção do professor de Matemática. 2007. Tese (Doutorado em Educação) - Universidade Federal do Rio Grande do Sul, Porto Alegre, 2007. Disponível em:

https://www.lume.ufrgs.br/handle/10183/13276. Acesso em: 04 dez. 2016.

LAVOR, João Ferreira de; ANDRIOLA, Wagner Bandeira; LIMA, Alberto Sampaio. Avaliando o impacto da qualidade da gestão acadêmica no desempenho dos cursos de graduação: um estudo em universidade pública brasileira. Revista Iberoamericana de Evaluación Educativa, Madri, v. 8, n. 2, p. 233-254, 2016. Disponível em: https://revistas.uam.es/index.php/riee/article/view/2887. Acesso em: 18 dez. 2016.

LIMA, Maria Cristina de Souza. Resultados de ingressantes no ensino superior via Enem: um ensaio de avaliação. 2011. Dissertação (Mestrado Profissional em Avaliação) - Fundação Cesgranrio, Rio de Janeiro, 2011. Disponível em:

http://mestrado.cesgranrio.org.br/pdf/dissertacoes2009/25\%20Abril\%202011\%20Dissertacao \%20Maria\%20Cristina\%20Souza\%20Lima\%20Turma\%202009.pdf. Acesso em: 30 nov. 2016.

LOPES, Fernanda Luzia; VENDRAMINI, Claudette Maria Medeiros. Propriedades psicométricas das provas de pedagogia do ENADE via TRI. Avaliação, Campinas; Sorocaba, v. 20, n. 1, p. 27-47, 2015. Disponível em:

http://submission.scielo.br/index.php/aval/article/view/104592. Acesso em: 25 nov. 2016.

LUCKESI, Cipriano. Avaliação da aprendizagem e educação. 2010. Disponível em: http://www.gestaoescolar.diaadia.pr.gov.br/arquivos/File/sem_pedagogica/fev_2010/avaliaca oaprendizagemluckesi.pdf. Acesso em: 6 maio. 2017.

MACHADO, Everton Zanini. Análise envoltória de dados sobre as universidades brasileiras: uma análise sobre eficiência. 2008. Dissertação (Mestrado em Economia) Pontifícia Universidade Católica do Rio Grande do Sul, Porto Alegre, 2008. Disponível em: http://tede2.pucrs.br/tede2/handle/tede/3858. Acesso em: 29 nov. 2016.

MOREIRA, Patrícia dos Santos Dias. A competência em informação nas bibliotecas escolares de Duque de Caxias - RJ: uma análise a partir do desempenho do Exame Nacional do Ensino Médio (Enem) de 2013. 2015. Monografia (Graduação em Biblioteconomia) Universidade Federal do Rio de Janeiro, Rio de Janeiro, 2015. Disponível em: https://pantheon.ufrj.br/handle/11422/355. Acesso em: 29 nov. 2016.

MORICONI, Gabriela Miranda; NASCIMENTO, Paulo Augusto Meyer Mattos. Fatores associados ao desempenho dos concluintes de Engenharia no Enade 2011. Revista Estudos em Avaliação Educacional, São Paulo, v. 25, n. 57, p. 248-278, 2014. Disponível em: http://publicacoes.fcc.org.br/ojs/index.php/eae/article/viewFile/2831/2704. Acesso em: 28 nov. 2016.

NOVOSSATE, Suzele. O Enade e os documentos curriculares: um estudo sobre a formação de professores de Biologia. 2010. Dissertação (Mestrado em Educação) Universidade Federal do Paraná, Curitiba, 2010. Disponível em: http://www.ppge.ufpr.br/teses/M10_Suzele\%20Novossate.pdf. Acesso em: 30 nov. 2016.

OLIVEIRA, Paulo Roberto de Faro. Convergências e divergências entre o desempenho no ENEM e no vestibular da UnB. Monografia (Graduação em Pedagogia) - Faculdade de Educação, Universidade de Brasília, Brasília, 2013. Disponível em: http://bdm.unb.br/handle/10483/6364. Acesso em: 03 dez. 2016.

OLIVEIRA, Susane Meira. Impacto do perfil dos professores na nota do Enade: uma análise dos cursos de Ciências Contábeis nas universidades federais brasileiras. 2014. 
Monografia (Graduação em Ciências Contábeis) - Faculdade de Administração, Ciências Contábeis e Econômicas, Universidade Federal de Goiás, Goiânia, 2014. Disponível em: https://repositorio.bc.ufg.br/bitstream/ri/11047/1/TCCG\%20-

$\% 20 \mathrm{Ci} \% \mathrm{C} 3 \%$ AAncias\%20Cont\%C3\%A1beis\%20-\%20Susane\%20Meira\%20Oliveira.pdf. Acesso em: 29 nov. 2016.

PARENTE, Nórlia Nabuco. As condições de acesso e permanência dos estudantes do curso de Licenciatura em Física do IFCE, campus de Sobral. 2014. Dissertação (Mestrado em Políticas Públicas e Gestão da Educação Superior) - Universidade Federal do Ceará, Fortaleza, 2014. Disponível em: http://www.repositorio.ufc.br/bitstream/riufc/10551/1/2014_dis_nnparente.pdf. Acesso em: 30 nov. 2016.

PIRES, Sanyo Drummond. Proposta de um modelo de equalização das provas do Enade de diferentes aplicações usando teste de ancoragem. 2013. Tese (Doutorado em Psicologia) - Universidade São Francisco, Itatiba, 2013. Disponível em: http://www.usf.edu.br/galeria/getImage/427/600070738556705.pdf. Acesso em: 29 nov. 2016.

PRIMI, Ricardo et al. Análise do funcionamento diferencial dos itens do Exame Nacional do Estudante (Enade) de Psicologia de 2006. Psico-USF, São Paulo, SP, v. 15, n. 3, p. 379-393, 2010. Disponível em: https://www.redalyc.org/pdf/4010/401036083011.pdf. Acesso em: 30 nov. 2016.

RODRIGUES, Ana Maria de Gois. Exame Nacional de Desempenho dos Estudantes -

Enade 2008: um estudo sobre o questionário de percepção sobre a prova de Pedagogia. 2013. Dissertação (Mestrado Profissional em Gestão e Avaliação da Educação Pública) -

Universidade Federal de Juiz de Fora, Juiz de Fora, 2013. Disponível em:

https://repositorio.ufjf.br/jspui/handle/ufjf/914. Acesso: 28 nov. 2016.

ROSSETTO, Cristina Botti de Souza. Oportunidades e desenvolvimento humano dos jovens no Brasil: três ensaios. 2016. Tese (Doutorado em Desenvolvimento Econômico) Universidade Federal do Paraná, Curitiba, 2016. Disponível em:

https://acervodigital.ufpr.br/handle/1884/43412. Acesso em: 04 dez. 2016.

SANTOS, Geisa Abreu Lira Corrêa dos; CONCORDIDO, Cláudia Ferreira Reis. Um estudo sobre o impacto das provas do Enem nas aulas de matemática. In: COLÓQUIO NTERNACIONAL EDUCAÇÃO, CIDADANIA E EXCLUSÃO: DIDÁTICA E AVALIAÇÃO, 4., 2015, Rio de Janeiro. Anais [...]. Rio de Janeiro: Universidade do Estado do Rio de Janeiro, 2015. Disponível em:

http://www.editorarealize.com.br/revistas/ceduce/trabalhos/TRABALHO_EV047_MD1_SA3 _ID1577_28052015163915.pdf. Acesso em: 28 nov. 2016.

SANTOS NETO, Agenor Sousa. Aplicação da modelagem de equações estruturais para avaliação da satisfação dos alunos de Engenharia de Produção de universidades privadas segundo o Enade 2011. 2016. Dissertação (Mestrado em Engenharia de Produção e Sistemas) - Universidade Católica de Goiás, Goiânia, 2016. Disponível em: http://tede2.pucgoias.edu.br:8080/handle/tede/2480\#preview-link0. Acesso em: 3 dez. 2016.

SCHWENGBER, Luciana. Exame Nacional de Desempenho de Estudantes: problematizando verdades sobre a formação do professor de Matemática. 2013. Dissertação (Mestrado em Educação) - Universidade de Santa Cruz do Sul, Santa Cruz do Sul, 2013. Disponível em: https://repositorio.unisc.br/jspui/handle/11624/553. Acesso em: 30 nov. 2016. 
SILVA, André Luiz. Desenvolvimento de um sistema on line de avaliação para análise do desempenho escolar: um estudo exploratório sobre avaliação em rede. 2015. Dissertação (Mestrado em Educação Escolar) - Universidade Estadual Paulista, Araraquara, SP, 2015. Disponível em: https://repositorio.unesp.br/handle/11449/123867. Acesso em: 29 nov. 2016.

SILVA, João Carlos da. Enade: um estudo sobre o desempenho das instituições de ensino superior nos anos de 2007 e 2010. Dissertação (Mestrado em Economia) - Pontifícia Universidade Católica de Brasília, Brasília, 2012. Disponível em:

https://bdtd.ucb.br:8443/jspui/handle/123456789/469. Acesso em: 28 nov. 2016.

SILVA, Leandro A.; MORINO, Anderson Hideki; SATO, Thiago Massahiro Conti. Prática de mineração de dados no Exame Nacional do Ensino Médio. In: WORKSHOPS DO

CONGRESSO BRASILEIRO DE INFORMÁTICA NA EDUCAÇÃO, 3., n. 1, p. 651-660,

2014, Dourados, MS. Anais [...]. Dourados, MS: Sociedade Brasileira de Computação, 2014. Disponível em: http://www.br-ie.org/pub/index.php/wcbie/article/view/3289. Acesso em: 22 abr. 2017.

SILVA, Mariana Cesar Verçosa; MELETTI, Silvia Márcia Ferreira. Avaliação em larga escala de alunos com necessidades educacionais especiais no município de Londrina (PR).

Revista Educação Especial, Santa Maria, RS, v. 25, p. 417-434, 2012. Disponível em: https://periodicos.ufsm.br/index.php/educacaoespecial/article/view/6531. Acesso em: 03 abr. 2017.

SILVA, Marjorie Cristina Rocha da; VENDRAMINI, Claudette Maria Medeiros; LOPES, Fernanda Luzia. Diferenças entre gênero e perfil sócio-econômico no Exame Nacional de Desempenho do Estudante. Avaliação, Campinas; Sorocaba, v. 15, n. 3, p. 185-202, 2010. Disponível em: http://www.scielo.br/scielo.php?pid=S141440772010000300010\&script=sci_abstract\&tlng=pt. Acesso em: 26 maio 2017.

SILVA, Simone Martins da. A avaliação em larga escala na Rede de Colégios Maristas RS. Dissertação (Mestrado em Educação) - Universidade do Vale do Rio dos Sinos, São Leopoldo, 2015. Disponível em: http://repositorio.jesuita.org.br/handle/UNISINOS/3816. Acesso em: 30 nov. 2016.

SILVA, Vanessa Ramos da. Enade e fluxo curricular nos cursos de graduação em Ciências Contábeis no Brasil. 2016. Dissertação (Mestrado em Ciências Contábeis) Universidade Federal de Uberlândia, Uberlândia, 2016. Disponível em: http://repositorio.ufu.br/handle/123456789/12633. Acesso em: 29 nov. 2016.

SILVEIRA, Priscilla Bacalhau Velloso. A provisão pública de ensino superior como mecanismo de seleção por habilidade: evidências para o Brasil. 2013. Dissertação (Mestrado em Economia de Empresas) - Fundação Getúlio Vargas, São Paulo, 2013. Disponível em: https://bibliotecadigital.fgv.br/dspace/handle/10438/10925. Acesso em: 03 dez. 2016.

SOARES, Delmiro. A baixa qualidade da educação no Brasil: quais fatores determinantes que interferem neste problema? Qual podemos priorizar para uma ação efetiva? 2014. Monografia (Graduação em Ciências Sociais) - Universidade Federal do Rio Grande do Sul, Porto Alegre, 2014. Disponível em: https://www.lume.ufrgs.br/handle/10183/105115. Acesso em: 30 nov. 2016.

SOUZA, Emerson Santana de. Enade 2006: determinantes do desempenho dos cursos de Ciências Contábeis. 2008. Dissertação (Mestrado em Ciências Contábeis) - Programa Multiinstitucional e Inter-Regional de Pós Graduação - Universidade de Brasília, Universidade Federal do Pernambuco e Universidade Federal do Rio Grande do Norte, 
Brasília, 2008. Disponível em: http://repositorio.unb.br/handle/10482/3949. Acesso em: 30 nov. 2016.

UNIVERSIDADE FEDERAL DE SÃO CARLOS. Departamento de Computação. Laboratório de Pesquisa em Engenharia de Software. StArt - State of the art through systematic reviews. 2013. Disponível em: http://lapes.dc.ufscar.br/tools/start_tool. Acesso em: 23 maio 2017.

VERHINE, Robert Evan; DANTAS, Lys Maria Vinhaes. Avaliação da educação superior no Brasil: do Provão ao ENADE. Ensaio: Avaliação e Políticas Públicas em Educação, Rio de Janeiro, v. 14, n. 52, p. 291-310, jul./set. 2006. Disponível em:

http://www.scielo.br/pdf/ensaio/v14n52/a02v1452.pdf. Acesso em: 03 abr. 2017.

VERHINE, Robert E.; DANTAS, Lys Maria Vinhaes. A avaliação do desempenho de alunos de educação Superior: uma análise a partir da experiência do Enade. In: LORDÊLO, José Albertino C.; DAZZANI, Maria Virgínia (org.).Avaliação educacional desatando e reatando nós. Salvador: EDUFBA, 2009. p. 173-199. Disponível em:

http://books.scielo.org/id/wd/pdf/lordelo-9788523209315.pdf\#page=174. Acesso em: 27 nov. 2016.

WALTENBERG, Fábio D.; CARVALHO, Márcia de. Cotas aumentam a diversidade dos estudantes sem comprometer o desempenho? Centro de Estudos sobre Desigualdade e Desenvolvimento, Rio de Janeiro, Texto para Discussão n. 73, mar. 2013. Disponível em: http://www.ie.ufrj.br/images/grupo_cede/publica\%C3\%A7\%C3\%B5es/site_antigo/tds/td73_9 438c.pdf. Acesso em: 4 dez. 2016. 